\title{
Effect of Electroconvulsive Therapy on Striatal Morphometry in Major Depressive Disorder
}

\author{
Benjamin SC Wade', Shantanu H Joshi ${ }^{2}$, Stephanie Njau' ${ }^{2}$, Amber M Leaver ${ }^{2}$, Megha Vasavada ${ }^{2}$, \\ Roger P Woods ${ }^{2,3}$, Boris A Gutman', Paul M Thompson', Randall Espinoza ${ }^{3}$ and Katherine L Narr ${ }^{*, 2,3}$ \\ 'Imaging Genetics Center, University of Southern California, Los Angeles, CA, USA; ${ }^{2}$ Ahmanson-Lovelace Brain Mapping Center, Department of \\ Neurology, University of California at Los Angeles, Los Angeles, CA, USA; ${ }^{3}$ Department of Psychiatry and Biobehavioral Sciences, University of \\ California at Los Angeles, Los Angeles, CA, USA
}

\begin{abstract}
Patients with major depression show reductions in striatal and paleostriatal volumes. The functional integrity and connectivity of these regions are also shown to change with antidepressant response. Electroconvulsive therapy (ECT) is a robust and rapidly acting treatment for severe depression. However, whether morphological changes in the dorsal and ventral striatum/pallidum relate to or predict therapeutic response to ECT is unknown. Using structural MRI, we assessed cross-sectional effects of diagnosis and longitudinal effects of ECT for volume and surface-based shape metrics of the caudate, putamen, pallidum, and nucleus accumbens in 53 depressed patients (mean age: 44.1 years, 13.8 SD; 52\% female) and 33 healthy controls (mean age: 39.3 years, 12.4 SD; 57\% female). Patients were assessed before ECT, after their second ECT, and after completing an ECT treatment index. Controls were evaluated at two time points. Support vector machines determined whether morphometric measures at baseline predicted ECT-related clinical response. Patients showed smaller baseline accumbens and pallidal volumes than controls $(P<0.05)$. Increases in left putamen volume $(P<0.03)$ occurred with ECT. Global increases in accumbens volume and local changes in pallidum and caudate volume occurred in patients defined as treatment responders. Morphometric changes were absent across time in controls. Baseline volume and shape metrics predicted overall response to ECT with up to $89 \%$ accuracy. Results support that ECT elicits structural plasticity in the dorsal and ventral striatum/pallidum. The morphometry of these structures, forming key components of limbic-cortical-striatal-pallidal-thalamic circuitry involved in mood and emotional regulation, may determine patients likely to benefit from treatment.
\end{abstract}

Neuropsychopharmacology (2016) 4I, 248I-249I; doi:I0.1038/npp.20 I6.48; published online 8 June 2016

\section{INTRODUCTION}

Major depression is one of the most common and economically burdensome psychiatric disorders (Kessler et al, 2003). Antidepressants and behavioral therapy are the most frequently prescribed treatments, but up to a third of patients remain unresponsive to initial treatment (Trivedi et al, 2006). With a rapid and high response rate, electroconvulsive therapy (ECT) is typically used after other failed treatments and can be particularly beneficial in suicidal, psychotic, or catatonic depression (Kellner et al, 2012). However, the neural mechanisms underlying clinical response to ECT remain uncertain. To advance therapeutic development, research providing clearer understanding of the neural correlates underlying successful antidepressant response is crucial.

Current theories describe depression as a brain network disorder. Converging evidence suggests neural disturbances

\footnotetext{
*Correspondence: Dr KL Narr, Ahmanson-Lovelace Brain Mapping Center, Department of Neurology, University of California Los Angeles, 935 Charles E Young Drive S \#225, Los Angeles, CA 90095, USA, Tel: + I 310267 51 19, Fax: I 310206 55 I8, E-mail: narr@ucla.edu Received 2 November 20 I5; revised I March 20 16; accepted 25 March 2016; accepted article preview online 12 April 2016
}

occur in a limbic-cortical-striatal-pallidal-thalamic circuit (Drevets et al, 2008). Structural alterations in the prefrontal cortex, particularly the anterior cingulate, and subcortical hippocampal, amygdalar, thalamic, and striatal/ pallidal centers are consistently implicated (Drevets et al, 2008; Lorenzetti et al, 2009; Schmaal et al, 2015). Lesion and functional imaging studies show these regions form networks governing mood regulation, reward sensitivity, and emotion (Koenigs and Grafman, 2009; Ochsner et al, 2012; Hamilton et al, 2013; Korgaonkar et al, 2013). Symptoms of depression such as amotivation, anhedonia, apathy, and rumination are linked to functional disturbances in the ventral striatum/ pallidum specifically (Disner et al, 2011; Ochsner et al, 2012; Kuhn et al, 2014). The ventral striatum, ventral pallidum, and continuity with the ventral caudate nucleus and putamen-basal ganglia regions with prominent limbic connections (Nieuwenhuys et al, 2008; Utter and Basso, 2008) - are themselves strongly interconnected, receive dopaminergic input from ventral midbrain regions (Haber and Knutson, 2010), and receive modulation by serotonergic midbrain pathways and other neurotransmitter systems (Di Matteo et al, 2008; Nieuwenhuys et al, 2008). The dorsal striatum, including the caudate nucleus, is involved in reward 
processing as relevant to depression symptomatology (Haber and Knutson, 2010).

Although less studied than the hippocampus and amygdala, basal ganglia substructures show lower volumes in meta-analyses of depression (Videbech, 1997; Koolschijn et al, 2009). Functional imaging studies demonstrate depressionrelated hyporesponsivity in striatal areas (Epstein et al, 2006; Smoski et al, 2009) and disturbances in fronto-striatal connectivity (Furman et al, 2011). Deficits in brain activation and functional connectivity in basal ganglia substructures are suggested to normalize with different antidepressant treatments (Stoy et al, 2012; Admon et al, 2015). In ECT-treated patients, we recently showed a hyperconnectivity between the ventral striatum and ventral default-mode network, while simultaneously demonstrating hypoconnectivity with the anterior default-mode network, patterns that were modulated by ECT (Leaver et al, 2015). These results suggest that the striatum is involved in functional desynchronization between dorsal and ventral cortico-limbic neural circuits in major depression and that normalization of these functional disturbances can occur with ECT.

Hippocampal connections with the hypothalamic-pituitary-adrenal axis, ventral striatal loop, and dopaminergic mesolimbic system appear particularly relevant to the pathophysiology of major depression (Russo and Nestler, 2013). We and other groups have shown that ECT elicits increases in hippocampal (Nordanskog et al, 2010; Tendolkar et al, 2013; Abbott et al, 2014; Joshi et al, 2015) and amygdala volume (Tendolkar et al, 2013; Joshi et al, 2015). However, whether structural plasticity occurs in the dorsal or ventral striatum/pallidum in association with treatment and illness recovery remains unknown. As full response to ECT may be observed in only 2-4 weeks (Kellner et al, 2012), using ECT as a treatment model may identify predictors and correlates of response over shorter time intervals where effects are expected to overlap with antidepressant therapies. We thus performed volumetric and shape-based analyses to investigate whether morphological changes are detectable before and after ECT in four constituents of the ventral basal ganglia: the caudate, putamen, pallidum, and nucleus accumbens. We compared patients with controls, and followed patients prospectively during an ECT treatment index series. Biomarkers predictive of treatment response could provide a major advance towards guiding clinical practice. We thus developed a machine-learning framework to determine whether morphometric differences, prior to treatment, might predict patients' responsiveness to ECT. On the basis of our observations of restoration of functional connectivity (Leaver et al, 2015), we predicted that ECT-related structural plasticity would also occur in ventral striatal and connected basal ganglia regions.

\section{MATERIALS AND METHODS}

\section{Subjects}

Participants included 53 patients experiencing a major depressive episode (28 females) recruited from individuals scheduled to receive ECT at the University of California, Los Angeles (UCLA) Resnick Neuropsychiatric Hospital. Diagnosis was determined by a board certified psychiatrist following Diagnostic Statistical Manual (DSM) IV-R criteria and additionally confirmed by the Mini-International Neuropsychiatric Interview (M.I.N.I.) (Sheehan et al, 1998). All patients had experienced two or more earlier major depressive episodes and failed to respond to at least two prior adequate medication trials in the index episode. Patients with comorbid psychiatric disorders including schizophrenia, schizoaffective disorders, post-traumatic stress disorder, attention hyperactive deficit disorder, and dissociative disorders were excluded. Forty-five patients were diagnosed with unipolar depression and 8 with bipolar disorder, though mania in the index episode was exclusionary. Other exclusion criteria included dementia, first-episode depression, onset after 50 years, depression related to serious medical illness, or any neuromodulation treatment (eg, vagal nerve stimulation, repetitive transcranial magnetic stimulation) within 6 months of the ECT index series. All patients were tapered off antidepressants and benzodiazepines in preparation for ECT and were completely free of medication for at least 48-72 $\mathrm{h}$ before enrollment and ECT treatment.

Patients were scanned at three time points: (i) baseline, prior to, and within $24 \mathrm{~h}$ of the initial ECT session (T1); (ii) after the second ECT session (T2, occurring 36-48 h after baseline assessment and before the third ECT); and (iii) within a week of completing the ECT treatment index series (T3, as individually determined based on stabilization of mood, approximately 4-6 weeks after first treatment). Of the 53 patients completing baseline, 45 completed T2 and 34 completed T3 assessments. Attrition was primarily due to early discontinuation of ECT, inability to come in for or to tolerate repeat scanning, and scanner hardware failures.

Thirty-three controls (19 females) with similar demographics to patient participants were recruited from the same geographical area using advertisements. Controls received M.I.N.I. (Sheehan et al, 1998) screening to exclude history of depression, other psychiatric, or medical illness, or a history of antidepressant use. Controls were scanned at two time points approximately 4-6 weeks apart. Additional exclusion criteria for all participants included history of alcohol or substance abuse within the past 6 months or dependence in the past 12 months, any neurological disorder, and contraindication to MRI scanning. Table 1 summarizes the demographic and clinical characteristics of our cohort. Subjects in this study overlap with those in two recent investigations of hippocampal and amygdala morphometry (Joshi et al, 2015) and functional connectivity (Leaver et al, 2015). All participants provided written informed consent as approved by the UCLA Institutional Review Board.

\section{ECT Treatment}

ECT (5000Q MECTA Corp.) was administered three times a week, using standard protocols for anesthesia (methohexital at $1 \mathrm{mg} / \mathrm{kg}$ dosage) and paralysis (succinylcholine at $1 \mathrm{mg} / \mathrm{kg}$ dosage). ECT followed the seizure threshold titration method: after establishing the seizure threshold, treatments were delivered at $5 \times$ seizure threshold for right unilateral d'Elia lead placement, using an ultra brief pulse-width $(0.3 \mathrm{~ms})$, and at $1.5 \times$ seizure threshold for bilateral placement, using a brief pulse-width $(0.5 \mathrm{~ms})$. 
Table I Demographic and Clinical Characteristics

\begin{tabular}{|c|c|c|}
\hline & $\begin{array}{l}\text { Patients, } \\
\mathrm{N}=53\end{array}$ & $\begin{array}{l}\text { Controls, } \\
N=33\end{array}$ \\
\hline Age, mean (SD), years & $44.1(13.8)$ & $39.3(12.4)$ \\
\hline Gender (M/F) & $25 / 28$ & $14 / 19$ \\
\hline \multicolumn{3}{|l|}{ Race/ethnicity } \\
\hline African American & 3 & 3 \\
\hline Asian & 4 & 3 \\
\hline Hispanic & 6 & 2 \\
\hline White & 38 & 24 \\
\hline Multi-ethnic & 1 & । \\
\hline Adjusted education, years & $15.78(2.70)$ & $16.94(2.30)$ \\
\hline Dextral/non-dextral ${ }^{a}$ & $37 / 12$ & $28 / 4$ \\
\hline \multicolumn{3}{|l|}{ Clinical Information } \\
\hline RUL/mixed lead placement/bilateral ${ }^{b}$ & $27 / 6 / 1$ & \\
\hline Unipolar/bipolar & $45 / 8$ & - \\
\hline Percent responders/non-responders ${ }^{c}$ & $65 / 35$ & \\
\hline Age at onset, mean (SD), years & $24.26(12.54)$ & - \\
\hline Current episode, mean (SD), years & $2.31(4.81)$ & - \\
\hline Lifetime illness, mean (SD), years & | $8.53(13.27)$ & - \\
\hline
\end{tabular}

\begin{tabular}{|c|c|c|}
\hline & $\begin{array}{l}\text { Responders, } \\
N=20\end{array}$ & $\begin{array}{c}\text { Non-responders, } \\
\mathrm{N}=14\end{array}$ \\
\hline Age, mean (SD), years & $44.9(12.6)$ & $37.2(14.6)$ \\
\hline Gender (M/F) & $12 / 8$ & $6 / 8$ \\
\hline \multicolumn{3}{|l|}{ Race/ethnicity } \\
\hline African American & 1 & I \\
\hline Asian & 1 & 3 \\
\hline Hispanic & 2 & 2 \\
\hline White & 15 & 8 \\
\hline Multi-ethnic & I & 0 \\
\hline Adjusted education, y & $10.1(2.4)$ & $9.6(2.9)$ \\
\hline \multicolumn{3}{|l|}{ Clinical Information } \\
\hline \# of ECT Index sessions, mean (SD) & $10.35(2.34)$ & I3.0 (3.53) \\
\hline \# of ECT Index sessions, range & $6-15$ & $8-22$ \\
\hline Unipolar/bipolar & $16 / 4$ & $12 / 2$ \\
\hline Age at onset, mean (SD), years & $26.84(14.64)$ & $20.07(10.94)$ \\
\hline Current episode, mean (SD), years & $1.94(3.04)$ & $2.52(3.63)$ \\
\hline Lifetime illness, mean (SD), years & $17.65(11.30)$ & $15.21(11.63)$ \\
\hline
\end{tabular}

\begin{tabular}{lccccc}
\hline Time point & TI, N=53 & T2, N=45 & T3, N=34 & $C 1, N=33$ & $C 2, N=31$ \\
\hline HAM-D & $24.34(6.16)^{\mathrm{d}}$ & $20.20(6.23)^{\mathrm{e}}$ & $12.46(8.04)^{\mathrm{f}}$ & - & - \\
QIDS-SR & $20.60(4.02)^{\mathrm{d}}$ & $16.75(5.47)^{\mathrm{e}}$ & $10.82(6.55)^{\mathrm{f}}$ & - & - \\
MADRS & $38.44(9.31)^{\mathrm{d}}$ & $31.47(9.55)^{\mathrm{e}}$ & $17.08(11.94)^{\mathrm{f}}$ & - & - \\
\hline
\end{tabular}

Abbreviations: $\mathrm{Cl}$ : control baseline; C2: control follow-up; HAM-D: Hamilton Rating Scale for Depression; MADRS: Montgomery-Åsberg Depression Rating Scale; QIDS-SR: Quick Inventory of Depressive Symptomatology - Self-Report; RUL: right unilateral lead placement; $\mathrm{TI}$ : patient baseline; $T 2$ : after the $2^{\text {nd }} \mathrm{ECT}$; T3: after the ECT index series.

a Handedness was estimated using the modified Edinburgh Handedness Inventory (Oldfield, 197I) where a laterality quotient of $<0.7$ defined non-dextrals.

bLead placement for patients completing all three time points.

${ }^{c}$ Response defined as $>50 \%$ improvement in HAM-D scores over the course of treatment.

'Significant effect between $\mathrm{TI}$ and $\mathrm{T} 2$.

eSignificant effect between T2 and T3.

fSignificant effect between $\mathrm{TI}$ and T3.

\section{Mood Ratings}

The Montgomery-Åsberg (MADRS) (Montgomery and Asberg, 1979), Hamilton (HAM-D-17) (Hamilton, 1960) depression rating scales and the Quick Inventory of
Depressive Symptomology Self Report (QIDS-SR) (Rush et al, 2003) were collected at the same time points as brain scanning. Patients were defined as treatment responders if they showed greater than $50 \%$ improvement in symptoms on the HAM-D. We additionally report differences in patients who responded with greater than $50 \%$ improvement across all mood scales.

\section{Image Acquisition}

High-resolution multi-echo T1-weighted MPRAGE images with real-time motion correction using navigators (Tisdall et al, 2012) were acquired on Siemens 3T Allegra (Erlangen, Germany) system (TEs/TR $=1.74,3.6,5.46,7.32 / 2530 \mathrm{~ms}$, $\mathrm{TI}=1260 \mathrm{~ms}, \quad \mathrm{FA}=7^{\circ}, \mathrm{FOV}=256 \times 256 \mathrm{~mm}, 192$ sagittal slices, voxel resolution $=1.3 \times 1.0 \times 1.0 \mathrm{~mm}^{3}$ ).

\section{Image Preprocessing and Segmentation}

Each T1-weighted image was processed and volumetrically quantified using the Freesurfer suite (version 5.3.0), which is documented and freely available online (http://surfer.nmr. mgh.harvard.edu/). Preprocessing steps included correction for magnetic field inhomogeneities, removal of non-brain tissue, and disconnection and segmentation of subcortical regions-of-interest including the bilateral putamen, pallidum, caudate, and nucleus accumbens in each subject and time point. All segmentations were visually inspected and manually corrected for minor topographic errors if needed. Intraclass correlation coefficients for each region from control subjects scanned 4-6 weeks apart indicated high concordance (accumbens $=0.77$; pallidum $=0.80$; putamen $=0.84$, and caudate $=0.93$ ). Figure 1 illustrates the locations of the caudate, putamen, pallidum, and accumbens.

\section{High-Resolution Surface-Based Analysis}

To investigate local group-level variation in subcortical anatomy, the segmented structures were transformed to a parameterized mesh surface using methods detailed in Gutman et al (2015). Briefly, each surface was conformally mapped to the spherical domain and rigidly rotated to a probabilistic atlas. Spherical Demons (Gutman et al, 2013) then non-linearly registered the spherical maps based on curvature profiles. Two surface-based functions were defined: (i) the global orientation function, defining the direction of the surface, and, (ii) the local thickness of the surface with respect to a skeletonized medial core. Spherical Demon was implemented using both the medial core and curvature to match each surface to the atlas. In this parameterization, two shape features are defined at each vertex: (i) radial distance (RD), a proxy for thickness, and (ii) the Jacobian determinant (JD), which indicates surface area dilation or contraction. The number of vertices for each surface scales with the average structure volume maintaining uniform resolution across structures (vertex counts: accumbens $=930$, caudate $=2502$, putamen $=2502$, and pallidum $=1254)$.

\section{Statistical Analysis}

Statistical analyses addressed (1) cross-sectional differences between patients and controls at baseline, (2) longitudinal 

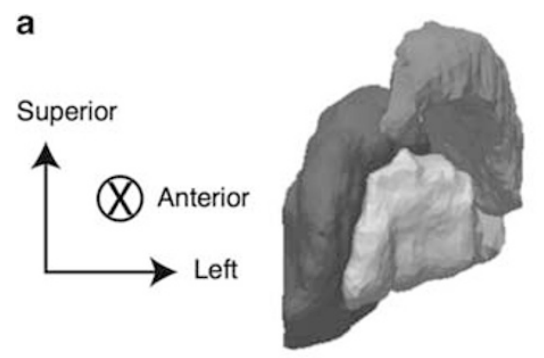

b

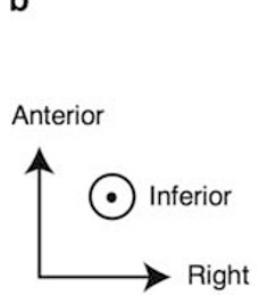

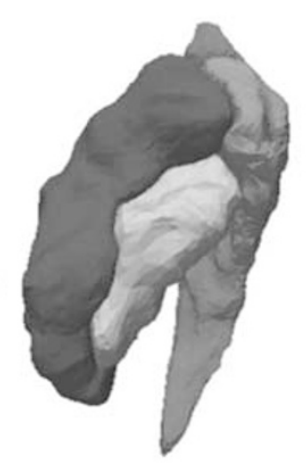
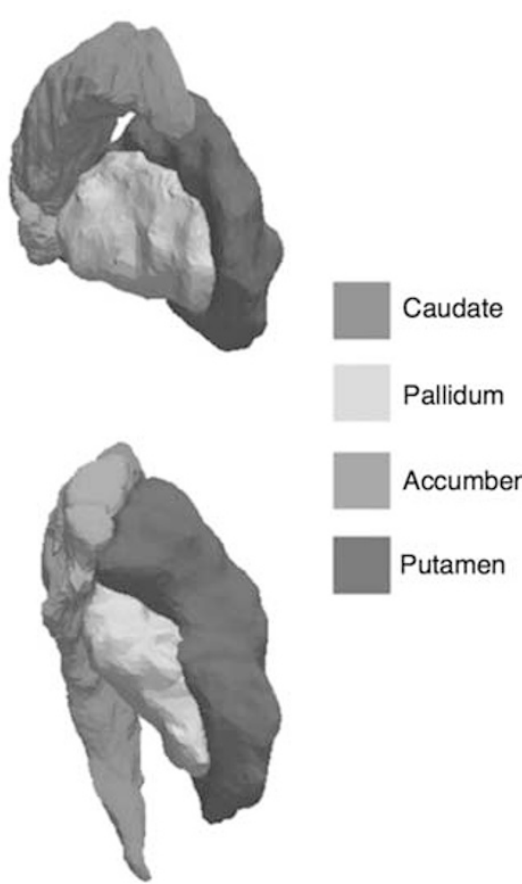

Accumbens Putamen

Figure I Relative anatomical locations of the putamen, pallidum, caudate, and nucleus accumbens as extracted from each MRI volume from (a) posterior and (b) inferior perspectives. All surface images are in radiological orientation, ie, left-right flipped. Orientation axes are provided for each subcortical map. Note that circles with central dots indicate the orientation coming out of the page while circles with central ' $x$ ' marks indicate the orientation going into the page.

effects of ECT (or time in control subjects), (3) crosssectional differences between ECT responders and nonresponders, and (4) predictors of ECT-related clinical response (using baseline imaging measures only). To reduce the number of comparisons while allowing for estimation of lateralized effects, hemisphere was included as a repeated measure in all volumetric analyses (implemented in IBM SPSS Statistics, v22). Shape-based analyses (executed in R; https://www.r-project.org) used the same statistical models except they were conducted in each hemisphere separately to allow for examination of focal effects.

Analysis \#1 above used the General Linear Model including sex, age, and brain volume as covariates to determine crosssectional effects of diagnosis (comparing baseline measures between patients and controls). Analysis \#2 used the General Linear Mixed Model, which models the correlation structure of repeated measurements while leveraging the statistical power gained using each subject as their own control, to determine longitudinal effects of ECT. These models, which allow for unbiased parameter estimates despite missing time points, included subject as a random effect and sex, age, and brain volume as covariates. Individual time points were compared pairwise for measures showing significant main effects of ECT. Analysis \#3 used the General Linear Model to determine whether change in morphometry over the course of ECT differed in treatment responders and non-responders. As difference scores (change in morphometric measures and change in mood scores) were examined, only patients completing all time points $(n=34)$ were examined. Age and sex were included as covariates.

To determine whether baseline morphometry predicts ECT response based on the combined and individual mood scales (examined separately), analysis \#4 used support vector machines (SVM), a supervised machine-learning algorithm. Leave-one-out cross validation was used to assess the performance of SVMs using a combined feature set of all baseline volume and shape measures. SVMs fit an optimal hyperplane in an n-dimensional feature space to separate labels matching input features. Our SVMs used radial kernels to model non-linear decision boundaries (Sánchez A, 2003). SVMs using radial kernels were compared with linear SVMs using DeLong's test for two receiver operating characteristic curves. Though the area under the curve (AUC) between the radial and linear SVMs was not significantly different, radial AUC was larger, thus the radial kernel was used. Grid search was used to find optimal combinations of the SVM cost and gamma parameters. Features were scaled to zero mean and unit variance. The significance of each SVM model was assessed using permutation tests achieved by comparing the observed AUC to a distribution of 1000 simulated AUCs derived from fitting SVMs to randomly shuffled labels. The proportion of permuted models with AUCs larger than the observed AUC provided the permutation $P$-value.

On the basis of our a priori hypotheses derived from prior findings showing ECT-related changes of functional connectivity in the striatum/pallidum with ECT (Leaver et al, 2015), a two-tailed alpha level of 0.05 was the threshold for significance for volumetric analyses. Surface-based analyses controlled for false positives using false discovery rate (Benjamini and Hochberg, 1995) using a 5\% false positive rate, surface-wise.

Finally, post hoc analyses were performed to test for differences between patients diagnosed with unipolar $(n=45)$ or bipolar depression $(n=8)$ and possible leadplacement effects (the \% of ECT sessions using right unilateral) for measures showing significant ECT effects. 

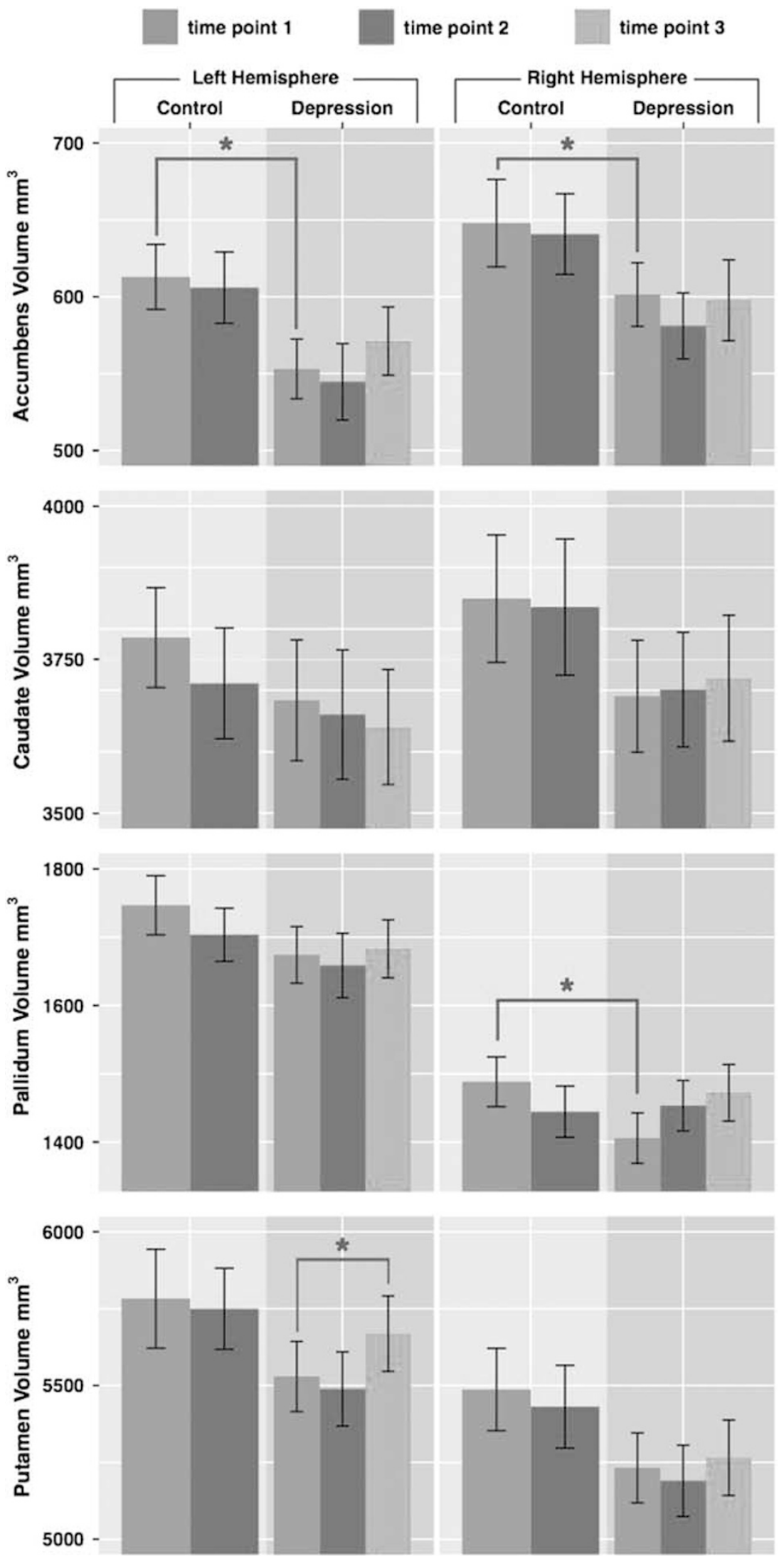

Figure 2 Average volume by group and time point for the accumbens, pallidum, and putamen. Error bars are $+/$ - I standard error. Asterisks (*) indicate significant differences. 
a

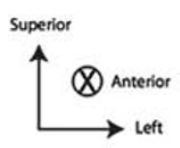

c

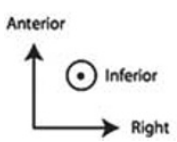

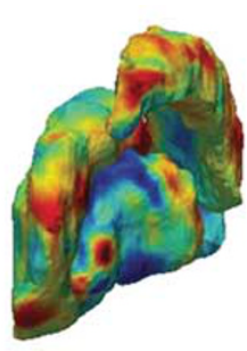
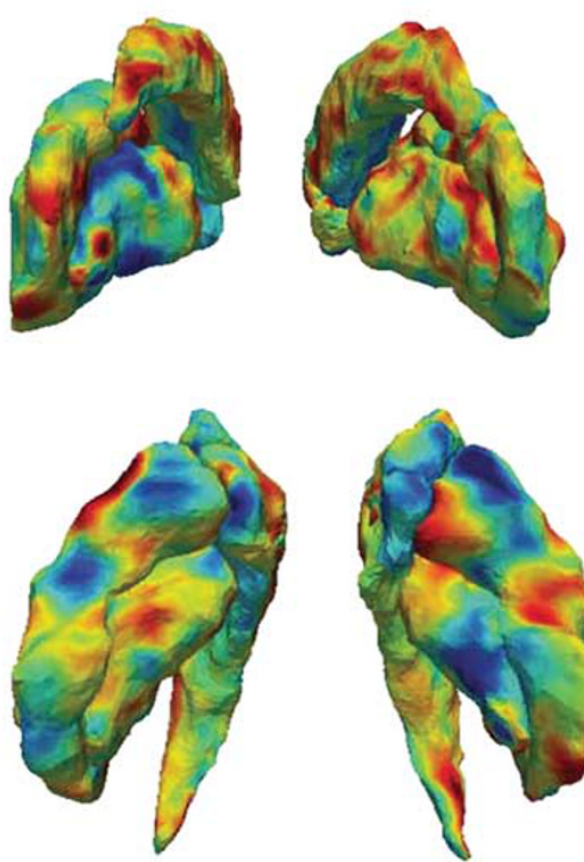

b

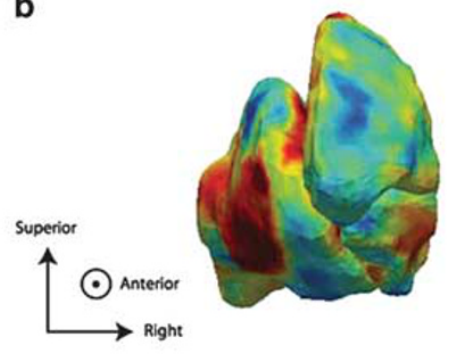

d

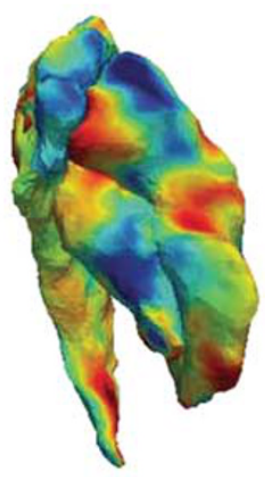

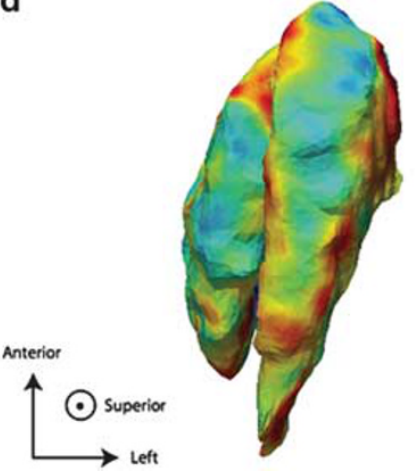

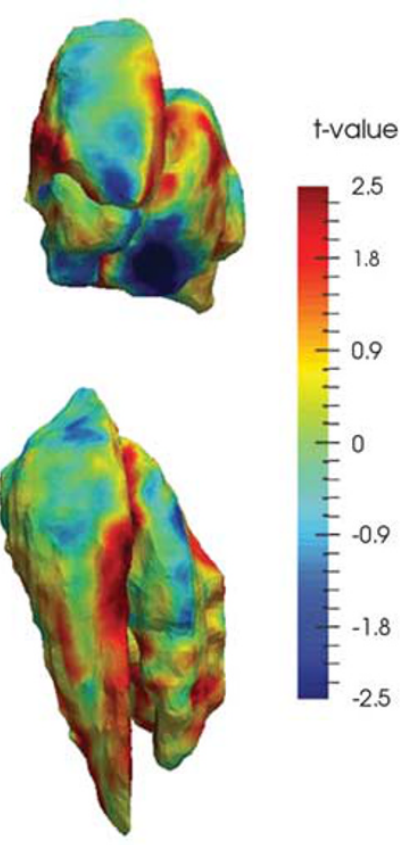

Figure 3 T-value maps of striatal and pallidal surfaces resulting from the mixed effects model examining the effect of ECT on thickness (RD, radial distance) over time. The perspectives are (a) posterior, (b) anterior, (c) inferior, and (d) superior views. All maps are in radiological orientation (ie, left-right flipped). Regions with warmer colors are expanding while those in cooler colors are contracting over time.

As lead placement was clinically determined (American Psychiatric Association. Committee on Electroconvulsive Therapy, 2001), effects of lead placement were examined while controlling for baseline mood scores.

\section{RESULTS}

\section{Demographic and Clinical Effects}

Sex, $X^{2}(1,85)=0.18, P=0.66$, and age, $F(1,85)=2.67$, $P=0.11$ distributions were similar in ECT patients and controls. MADRS, HAM-D, and QIDS rating scales showed highly significant effects of ECT, $\mathrm{F}(2,36.75)=38.66$, $\mathrm{F}(2,38.16)=32.74, \mathrm{~F}(2,36.81)=37.60$; all $P<0.0001$, respectively. Demographic and clinical details for each group and time point are provided in Table 1.

\section{Cross-Sectional Effects of Diagnosis}

Volumetric analyses showed smaller volumes of the nucleus accumbens, $\mathrm{F}(1,81)=4.67, P=0.034,-8.0 \%$ and pallidum, $\mathrm{F}(1,81)=3.96, P=0.05,-4.9 \%$ in patients relative to controls at baseline. Cross-sectional effects were nonsignificant for the putamen, $\mathrm{F}(1,82.04)=2.76, P=0.101$ and caudate $\mathrm{F}(1,80.70)=0.095, P=0.76$ and no region showed hemispheric interactions. Means for each diagnostic group and time point are plotted in Figure 2. No significant between-group shape variation was observed.

\section{Longitudinal Effects of ECT}

A significant volumetric association with ECT, $\mathrm{F}(2,42.80)=$ 5.36, $P=0.008$ and ECT by hemisphere interaction was observed for the putamen, $\mathrm{F}(1,44.17)=3.40, P=0.042$. A significant increase in left putamen volume occurred with ECT, $\mathrm{F}(1,39.83)=3.57, P=0.038,2.4 \%$. Pairwise comparisons of time points revealed significant volume increases between T1 and T3, P=0.01 (Figure 2). Regional shape changes were non-significant for all four substructures, though statistical maps, shown in Figure 3, suggest more diffuse surface expansion with ECT. Effects of time in control subjects were non-significant for both shape and volume.

\section{Effects of Morphometric Change with Clinical Response}

Significant effects of response status were observed for the volume of the accumbens, $\mathrm{F}(1,28)=4.29, P=0.048$ where responders showed volumetric gain, $F(2,18.98)=9.18$, $P=0.002$, with no change in non-responders $(P>0.05)$. There was a significant effect of response status for change in caudate volume over time, $\mathrm{F}(1,29.03)=4.29, \quad P=0.048$. Though volumes tended to increase over time in responders and decrease over time in non-responders, these effects were non-significant within group (both $P>0.05$ ). Significant regional increases in caudate morphometry were observed with the JD of the left superior and inferior caudate increasing in HAM-D responsive patients and decreasing in non-responsive patients over time. Finally, local decreases in the posterior medial aspect of the right pallidum for $\mathrm{RD}$ and JD were present in responders at time point 3 . Figure 4 shows significant volume and shape differences by ECT-response.

\section{Baseline Predictive Effects}

Using the entire combined set of baseline shape and volume features, SVM prediction of clinical outcome using the 
combined mood scale resulted in 90\% AUC (95\% CI: $68-93 \%, P=0.000$ ), a maximum accuracy of $89 \%$. Response prediction for individual mood scales yielded 54\% AUC (95\% CI 29-78\%; $P=0.599$, maximum accuracy $=72 \%$ ) for HAM-D; 59\% AUC (95\% CI 36-80\%; $P=0.392$, maximum accuracy $=68 \%)$ for MADRS, and $84 \%$ AUC (95\% CI 64-93\%; $P=0.001$, maximum accuracy $=75 \%$ ) for QIDS. Figure $5 \mathrm{a}$ plots the receiver operating characteristic curves associated with each SVM while Figure 5b plots the associated accuracy, sensitivity, and specificity values across a range of decision thresholds. As a post hoc analysis, we investigated the performance of SVMs using metric-specific features in the prediction of response. With performances averaged across mood scales, RD-specific features yielded a mean of $77 \%$, JD $63 \%$, and volume $60 \%$ AUC. Because overfitting with high-dimensional features is a common problem, we tested whether LASSO feature selection, implemented within each cross-validation fold, would significantly affect classifier performance. LASSO retained an average of two features and provided an AUC of $76 \%$, which did not significantly differ from the full-feature SVM $(P=0.226)$.

\section{Post hoc Analyses of Diagnostic Category and ECT Lead Placement}

There were no significant differences for volume or shape when comparing unipolar $v s$ bipolar depression. Associations with the proportion of right unilateral treatments given across the ECT index series also failed to show significant effects for volume or shape.

\section{DISCUSSION}

Our study addressed whether ECT impacts the morphometry of four components of the dorsal and ventral basal ganglia that are known to have a role in cognitive and affective function of high relevance to depression: the accumbens, putamen, pallidum, and caudate. Each structure's morphometry was modeled using global volumetric and local descriptions of shape variation. Exploring effects of diagnosis and treatment, we contrasted region-of-interest morphometry between patients and controls at baseline, in patients across the course of ECT, and between patient responders and non-responders. Finally, we showed that patients' responsiveness to ECT could be predicted from baseline brain morphometry using machine learning.

Cross-sectionally, we observed reduced accumbens and pallidum volumes in patients prior to treatment. This corroborates prior reports of lower striatal volumes in depressed cohorts (Videbech, 1997; Koolschijn et al, 2009). We did not detect shape differences between patients and controls, so volumetric differences may be more globally dispersed than focal. Several longitudinal patterns emerged. The left putamen volume increased between baseline and the end of the ECT treatment index. We did not observe significant ECT effects for morphometry in other regions-ofinterest. Differential patterns of morphometry were observed within patients based on their responsiveness to ECT. The accumbens volume significantly increased over the course of ECT (Figure 4a). However, effects in the right medial pallidum suggested more localized volume reductions in responders.

Cumulatively, the above findings support that structural deficits in striatal and paleostriatal structures occur in major depression and ECT leads to neuroplasticity in these regions. While the basal ganglia have pivotal roles in psychomotor function, these regions are also densely connected with proximal limbic regions including the hippocampus and amygdala (Nieuwenhuys et al, 2008). As there is typically a lengthy delay between the initiation of standard antidepressant therapies and clinical response, biomarkers that help guide future treatment decisions may have substantial clinical impact. Using SVMs to learn differential patterns of brain morphometry between responsive and non-responsive patients, we predicted patient response with up to $89 \%$ accuracy when defining response across all mood scales. Some demographic and clinical factors, such as the presence of psychotic symptoms, have been associated with improved ECT response (Petrides et al, 2001). Yet, without any other basis for determining potential outcome, an $89 \%$ predictive value may be very beneficial, particularly if this prediction generalizes to other forms of antidepressant treatment.

ECT promoted a volumetric increase or local surface deformations in the striatum over short time intervals (4-6 weeks). A leading hypothesis regarding the mechanisms underlying major depression is that neurotrophic factors are adversely affected. This is supported by observations that antidepressant treatments influence transduction pathways associated with neuronal plasticity (Duman, 2002). For example, electroshock-the animal model of ECT-stimulates neurogenesis in the hippocampus of rodents (Malberg et al, 2000) and non-human primates (Perera et al, 2007). As adult neurogenesis occurs in the hippocampal dentate gyrus, in line with a neurotrophic model of antidepressant response (Kellner et al, 2012; Fosse and Read, 2013), several prior structural imaging studies of ECT in humans showed ECTrelated increases in hippocampal volume (Duman, 2002; Nordanskog et al, 2010; Tendolkar et al, 2013; Abbott et al, 2014; Jorgensen et al, 2015). In a sample overlapping with the current study, we also observed changes in hippocampal morphometry with ECT and relationships with symptom improvement (Joshi et al, 2015). Notably, Inta and Gass (2015) reported ECT-triggered neurogenesis in striatal and frontal brain regions following ECT in a rat model suggesting that cell proliferation extends beyond the hippocampus. Others (Liu et al, 2009) have also reported increased subventricular zone neuroblast cell proliferation to striatal areas following seizures. Further, ECT-related structural neuroplasticity is shown in the amygdala (Tendolkar et al, 2013; Joshi et al, 2015). These subcortical limbic structures are intricately connected to the striatum, and via the cortico-striatal-pallido-thalamo-cortical loop and projections to the hypothalamus and brainstem, act together to influence emotional expression and motivation (Cardinal et al, 2002).

Additional mechanisms may account for ECT-related neuroplasticity. These include an increase in monoamine neurotransmitter production, release of hypothalamic hormones, increased angiogenesis (Wennstrom et al, 2006), glial cell activation (Jansson et al, 2009), and gliogenesis (Wennstrom et al, 2006). The ventral striatum and parts of the dorsal striatum form part of the mesolimbic dopaminergic pathway involved in reward processing. Patients with 
depression frequently show a diminished ability to derive pleasure or positive motivation from rewarding stimuli leading to symptoms of anhedonia (Zhang et al, 2013).
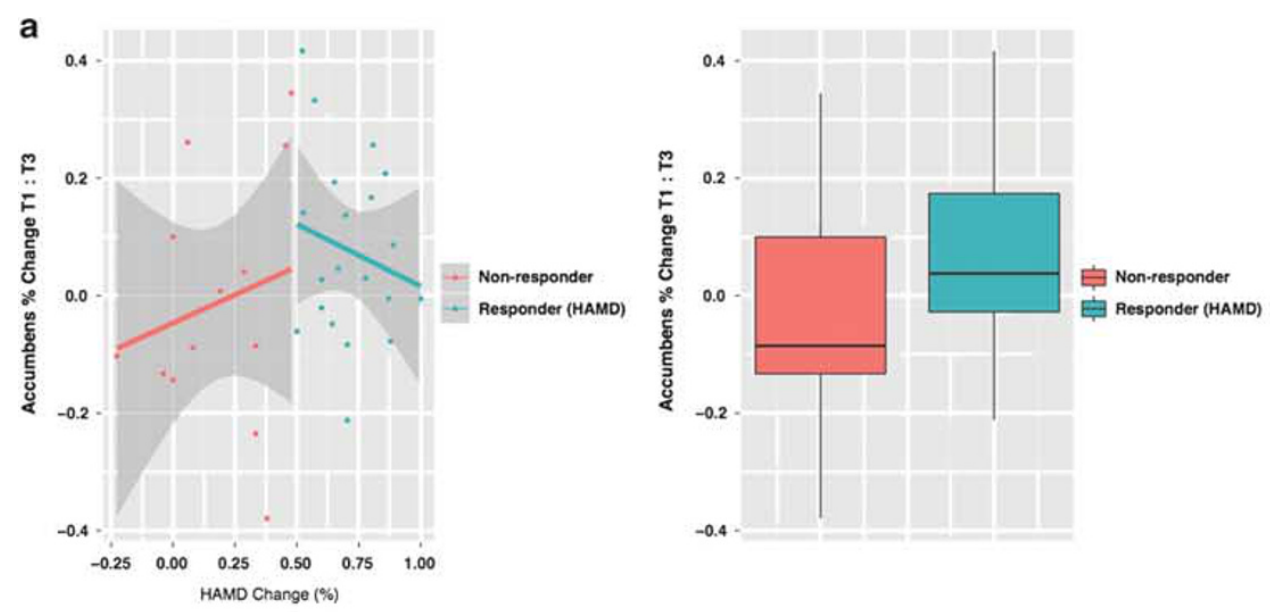

b
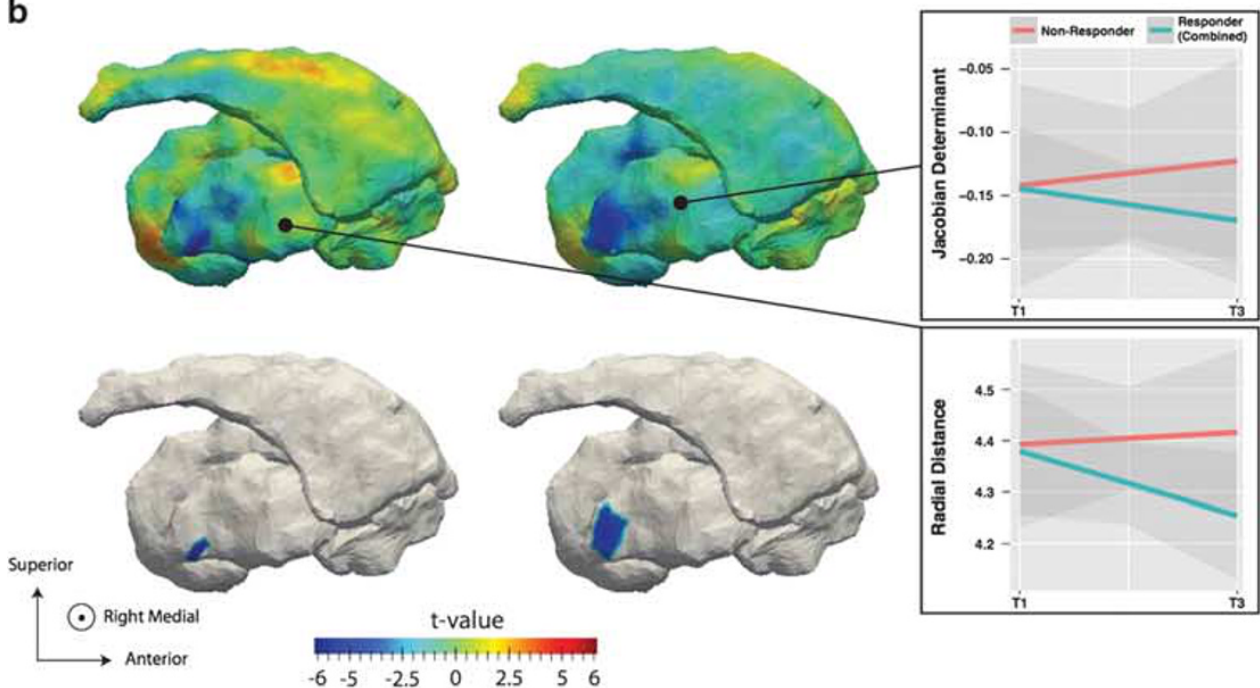

c
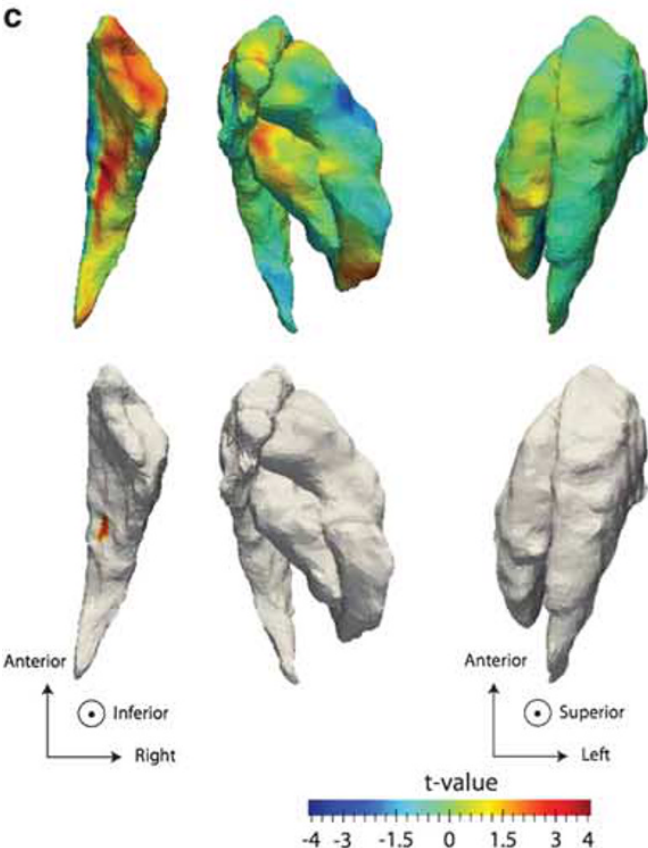

Disease-related abnormalities in reward processing are characterized as reflecting 'reward hyposensitivity', and brain activation studies typically show hypoactivation in striatal 
and prefrontal regions (Forbes et al, 2009; Zhang et al, 2013). Human neuroimaging studies also demonstrate that disturbed regulation between regions involved in reward processing occur via the serotonin transporter (Hahn et al, 2014). Preclinical studies further show that anxiety and depressive-like behavior associate with lower levels of serotonin and norepinephrine in the striatum (Brenes et al, 2008) where serotonergic and adrenergic systems are targets of commonly prescribed antidepressants. Though it is possible that changes in striatal morphometry may be influenced by anesthesia rather than by treatment-related effects, observations of change in responders $v s$ nonresponders makes this less likely.

Because shape-based descriptors are less commonly used than volumetric descriptions of brain structures, we endeavored to demonstrate their value as both predictors in the SVM and a descriptor of morphometry. In the context of classification, we compared the use of RD-only, JD-only, and volume-only features and showed the average performance of SVMs using shape-based descriptors was higher than those using volume-only. Overfitting is another concern when using high-dimensional features. Thus, we compared an SVM using all features with a LASSO subset. AUC did not significantly differ between these models, therefore it is unlikely that significant overfitting occurred with the full feature set; however, this may be confirmed with a larger sample.

The value of shape-based approaches is their ability to reveal local variation in surface topology rather than simply identify gross volumetric variation. This is particularly valuable because we did not segment sub-nuclei of subcortical structures. The locality of the shape measures could thus inform future studies using subfield measurements. A related concern is that shape and volume are highly correlated. Indeed, the average correlation between $\mathrm{RD}$ and volume was $r=0.37$ and JD and volume $r=0.44$; however, this is expected because the measures are of the same structure. The value of the descriptors' combined use lies in their ability to reveal separate, complementary aspects of the structure's topology.

Placed in the context of existing literature, ECT-related structural neuroplasticity in basal ganglia substructures could be attributed to neurotrophic factors that include cell and/or synaptic proliferation possibly linked to changes in monoaminergic neurotransmitter systems that form part of the prefrontal-striatal-limbic circuitry. Though this is the first study to our knowledge to show that ECT affects the morphometry of the striatum and paleostriatum, several
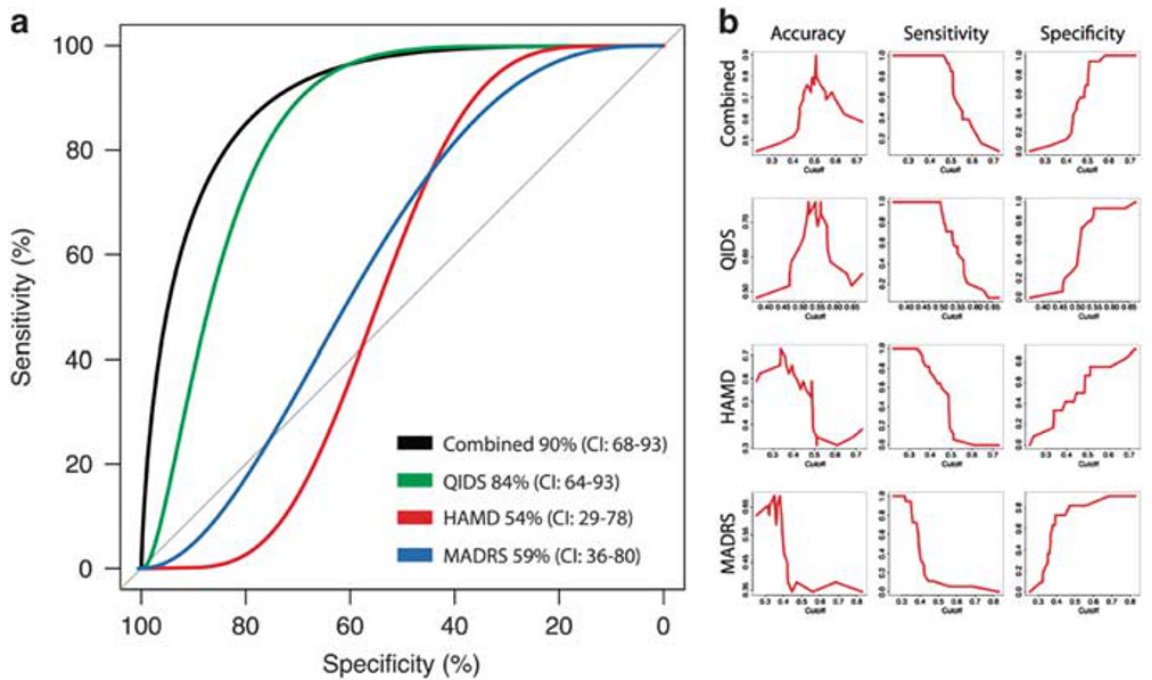

Figure 5 (a) Receiver operating characteristic curves resulting from support vector machines prediction of responsive patients from baseline shape and volume features. One curve is given for each mood scale (Combined, QIDS-SR, HAM-D, and MADRS) used to determine response status. (b) Plots of the accuracy, sensitivity, and specificity values mapped separately for each model across a range of decision thresholds.

Figure 4 (a, left) Scatterplot of the percent change in bilateral nucleus accumbens volume between baseline and the end of the ECT index (TI and T3) and the percent improvement in patients defined as ECT responders and non-responders on the HAM-D scale. This shows responders having generally larger gains in accumbens volume than non-responders. However, within responder and non-responder groups, scatterplots showing change in volume with change in mood scale show slopes in the unexpected direction, though these are non-significant. (a, right) Boxplot of the percent change in bilateral nucleus accumbens volume by response category showing larger average change in the HAM-D responders relative to non-responders. (b) Uncorrected (top) and FDR-thresholded (bottom) t-value maps of shape variation at time point 3 by combined-scale response status for the right pallidum. Negative t-values indicate smaller values in responders, whereas positive t-values indicate larger values in responders. The left column shows RD maps and right column shows JD maps. Linear regression trend lines with 95\% confidence intervals color-coded by response status are shown for the average shape feature (RD or JD) within the right pallidum. (c) Maps of the longitudinal association of the surface area (JD measure) with response status. The top maps are uncorrected t-maps while the bottom maps are FDR-thresholded. Positive t-values indicate an increased rate of surface area gain in patients responsive to ECT relative to non-responsive patients. The associated interaction plots show the significant differential trajectories of the average surface area of the left caudate between responders and non-responders. 
limitations exist. First, despite a priori hypotheses justifying the study of these regions, these findings warrant independent replication. As morphometry of subcortical regions are highly variable even in normal populations, this variability may have impacted our ability to detect local shape changes. Finally, though we investigated relationships with changes in overall symptom ratings in the current study, it is possible that ECT-related neuroplasticity may be more closely related to specific symptoms such as anhedonia, apathy, amotivation, and rumination as have been linked with altered striatal circuitry in functional imaging studies.

\section{CONCLUSION}

Our findings provide new evidence to support that ECT induces neuroplastic changes in striatal regions in addition to other subcortical limbic regions. We also demonstrate that these changes are associated with clinical improvement and have the potential to predict an affected individual's treatment response using machine learning based solely on imaging features.

\section{FUNDING AND DISCLOSURE}

The authors declare no conflict of interest.

\section{ACKNOWLEDGMENTS}

Award Number R01MH092301 from the National Institute of Mental Health supported this study. This work is also supported in part by NIH 'Big Data to Knowledge' (BD2K) Center of Excellence grant U54 EB020403, funded by a cross$\mathrm{NIH}$ consortium including NIBIB and NCI and by the National Science Foundation Graduate Research Fellowship under Grant No. DGE-0707424.

\section{REFERENCES}

Abbott CC, Jones T, Lemke NT, Gallegos P, McClintock SM, Mayer AR et al (2014). Hippocampal structural and functional changes associated with electroconvulsive therapy response. Transl Psychiatry 4: e483.

Admon R, Nickerson LD, Dillon DG, Holmes AJ, Bogdan R, Kumar P et al (2015). Dissociable cortico-striatal connectivity abnormalities in major depression in response to monetary gains and penalties. Psychol Med 45: 121-131.

American Psychiatric Association. Committee on Electroconvulsive Therapy, Weiner RD (2001). The Practice of Electroconvulsive Therapy: Recommendations for Treatment, Training, and Privileging: A Task Force Report of the American Psychiatric Association, 2nd edn. American Psychiatric Association: Washington, D.C., USA. viii, 355.

Benjamini Y, Hochberg Y (1995). Controlling the false discovery rate: a practical and powerful approach to multiple testing. $J$ R Stat Soc Series B 57: 289-300.

Brenes JC, Rodriguez O, Fornaguera J (2008). Differential effect of environment enrichment and social isolation on depressive-like behavior, spontaneous activity and serotonin and norepinephrine concentration in prefrontal cortex and ventral striatum. Pharmacol Biochem Behav 89: 85-93.

Cardinal RN, Parkinson JA, Hall J, Everitt BJ (2002). Emotion and motivation: the role of the amygdala, ventral striatum, and prefrontal cortex. Neurosci Biobehav Rev 26: 321-352.
Di Matteo V, Pierucci M, Esposito E, Crescimanno G, Benigno A, Di Giovanni G (2008). Serotonin modulation of the basal ganglia circuitry: therapeutic implication for Parkinson's disease and other motor disorders. Prog Brain Res 172: 423-463.

Disner SG, Beevers CG, Haigh EA, Beck AT (2011). Neural mechanisms of the cognitive model of depression. Nat Rev Neurosci 12: 467-477.

Drevets WC, Price Jl Fau - Furey ML, Furey ML (2008). Brain structural and functional abnormalities in mood disorders: implications for neurocircuitry models of depression. Brain Struct Funct 213: 93-118.

Duman RS (2002). Pathophysiology of depression: the concept of synaptic plasticity. Eur Psychiatry 17(Suppl 3): 306-310.

Epstein J, Pan H, Kocsis JH, Yang Y, Butler T, Chusid J et al (2006). Lack of ventral striatal response to positive stimuli in depressed versus normal subjects. Am J Psychiatry 163: 1784-1790.

Forbes EE, Hariri AR, Martin SL, Silk JS, Moyles DL, Fisher PM et al (2009). Altered striatal activation predicting real-world positive affect in adolescent major depressive disorder. Am J Psychiatry 166: 64-73.

Fosse R, Read J (2013). Electroconvulsive treatment: hypotheses about mechanisms of action. Front Psychiatry 4: 94.

Furman DJ, Hamilton JP, Gotlib IH (2011). Frontostriatal functional connectivity in major depressive disorder. Biol Mood Anxiety Disord 1: 11-11.

Gutman B, Jahanshad N, Ching C, Wang Y, Kochunov P, Nichols T et al (2015). Medial demons registration localizes the degree of genetic influence over subcortical shape variability: an $\mathrm{N}=1480$ meta-analysis. Proc IEEE Int Symp Biomed Imaging 2015: 1402-1406.

Gutman BA, Madsen SK, Toga AW, Thompson PM. A family of fast sphericalregistration algorithms for cortical shapes. In: Multimodal Brain Image Analysis. Volume 8159. Springer International Publishing, 2013, pp 246-257.

Haber SN, Knutson B (2010). The reward circuit: linking primate anatomy and human imaging. Neuropsychopharmacology 35: $4-26$.

Hahn A, Haeusler D, Kraus C, Hoflich AS, Kranz GS, Baldinger P et al (2014). Attenuated serotonin transporter association between dorsal raphe and ventral striatum in major depression. Hum Brain Mapp 35: 3857-3866.

Hamilton JP, Chen MC, Gotlib IH (2013). Neural systems approaches to understanding major depressive disorder: an intrinsic functional organization perspective. Neurobiol Dis 52: $4-11$.

Hamilton M (1960). A rating scale for depression. J Neurol Neurosurg Psychiatry 23: 56-62.

Inta D, Gass P (2015). Is forebrain neurogenesis a potential repair mechanism after stroke? J Cereb Blood Flow Metab 35: 1220-1221.

Jansson L, Wennstrom M, Johanson A, Tingstrom A (2009). Glial cell activation in response to electroconvulsive seizures. Prog Neuropsychopharmacol Biol Psychiatry 33: 1119-1128.

Jorgensen A, Magnusson P, Hanson LG, Kirkegaard T, Benveniste H, Lee $\mathrm{H}$ et al (2015). Regional brain volumes, diffusivity, and metabolite changes after electroconvulsive therapy for severe depression. Acta Psychia Scand (e-pub ahead of print 3 July 2015).

Joshi SH, Espinoza RT, Pirnia T, Shi J, Wang Y, Ayers B et al (2015). Structural plasticity of the hippocampus and amygdala induced by electroconvulsive therapy in major depression. Biol Psychiatry 79: 282-292.

Kellner CH, Greenberg RM, Murrough JW, Bryson EO, Briggs MC, Pasculli RM (2012). ECT in treatment-resistant depression. Am J Psychiatry 169: 1238-1244.

Kessler RC, Berglund P, Demler O, Jin R, Koretz D, Merikangas KR et al (2003). The epidemiology of major depressive disorder: Results from the national comorbidity survey replication. JAMA 289: 3095-3105. 
Koenigs M, Grafman J (2009). The functional neuroanatomy of depression: distinct roles for ventromedial and dorsolateral prefrontal cortex. Behav Brain Res 201: 239-243.

Koolschijn PC, van Haren NE, Lensvelt-Mulders GJ, Hulshoff Pol HE, Kahn RS (2009). Brain volume abnormalities in major depressive disorder: a meta-analysis of magnetic resonance imaging studies. Hum Brain Mapp 30: 3719-3735.

Korgaonkar MS, Grieve SM, Etkin A, Koslow SH, Williams LM (2013). Using standardized fMRI protocols to identify patterns of prefrontal circuit dysregulation that are common and specific to cognitive and emotional tasks in major depressive disorder: first wave results from the iSPOT-D study. Neuropsychopharmacology 38: 863-871.

Kuhn S, Vanderhasselt MA, De Raedt R, Gallinat J (2014). The neural basis of unwanted thoughts during resting state. Soc Cogn Affect Neurosci 9: 1320-1324.

Leaver AM, Espinoza R, Joshi SH, Vasavada M, Njau S, Woods RP et al (e-pub ahead of print 22 September 2015). Desynchronization and plasticity of striato-frontal connectivity in major depressive disorder. Cereb Cortex.

Liu F, You Y, Li X, Ma T, Nie Y, Wei B et al (2009). Brain injury does not alter the intrinsic differentiation potential of adult neuroblasts. J Neurosci 29: 5075-5087.

Lorenzetti V, Allen NB, Fornito A, Yucel M (2009). Structural brain abnormalities in major depressive disorder: A selective review of recent MRI studies. J Affect Disord 117: 1-17.

Malberg JE, Eisch AJ, Nestler EJ, Duman RS (2000). Chronic antidepressant treatment increases neurogenesis in adult rat hippocampus. J Neurosci 20: 9104-9110.

Montgomery SA, Asberg M (1979). A new depression scale designed to be sensitive to change. Br J Psychiatry 134: 382-389.

Nieuwenhuys R, Voogd J, Huijzen Cv (2008). The Human Central Nervous System. Springer Science \& Business Media (ed), 4th edn. Springer: Berlin, NY, USA. xiv, 967.

Nordanskog P, Dahlstrand U, Larsson MR, Larsson EM, Knutsson L, Johanson A (2010). Increase in hippocampal volume after electroconvulsive therapy in patients with depression: a volumetric magnetic resonance imaging study. J ECT 26: 62-67.

Ochsner KN, Silvers JA, Buhle JT (2012). Functional imaging studies of emotion regulation: a synthetic review and evolving model of the cognitive control of emotion. Ann N Y Acad Sci 1251: E1-E24.

Oldfield RC (1971). The Assessment and Analysis of Handedness: the Edinburgh Inventory. Neuropsychologia 9: 97-113.

Perera TD, Coplan JD, Lisanby SH, Lipira CM, Arif M, Carpio C et al (2007). Antidepressant-induced neurogenesis in the hippocampus of adult nonhuman primates. J Neurosci 27: 4894-4901.

Petrides G, Fink M, Husain MM, Knapp RG, Rush AJ, Mueller M et al (2001). ECT remission rates in psychotic versus nonpsychotic depressed patients: a report from CORE. J ECT 17: 244-253.
Rush AJ, Trivedi MH, Ibrahim HM, Carmody TJ, Arnow B, Klein DN et al (2003). The 16-Item Quick Inventory of Depressive Symptomatology (QIDS), clinician rating (QIDS-C), and self-report (QIDS-SR): a psychometric evaluation in patients with chronic major depression. Biol Psychiatry 54: 573-583.

Russo SJ, Nestler EJ (2013). The brain reward circuitry in mood disorders. Nat Rev Neurosci 14: 609-625.

Sánchez A VD (2003). Advanced support vector machines and kernel methods. Neurocomputing 55(1-2): 5-20.

Schmaal L, Veltman DJ, van Erp TG, Samann PG, Frodl T, Jahanshad $\mathrm{N}$ et al (2015). Subcortical brain alterations in major depressive disorder: findings from the ENIGMA Major Depressive Disorder working group. Mol Psychiatry (e-pub ahead of print 30 June 2015).

Sheehan DV, Lecrubier Y, Sheehan KH, Amorim P, Janavs J, Weiller E et al (1998). The Mini-International Neuropsychiatric Interview (M.I.N.I.): the development and validation of a structured diagnostic psychiatric interview for DSM-IV and ICD-10. J Clin Psychiatry 59(Suppl 20): 22-33; quiz 34-57.

Smoski MJ, Felder J, Bizzell J, Green SR, Ernst M, Lynch TR et al (2009). fMRI of alterations in reward selection, anticipation, and feedback in major depressive disorder. J Affect Disord 118: 69-78.

Stoy M, Schlagenhauf F, Sterzer P, Bermpohl F, Hagele C, Suchotzki $\mathrm{K}$ et al (2012). Hyporeactivity of ventral striatum towards incentive stimuli in unmedicated depressed patients normalizes after treatment with escitalopram. J Psychopharmacol 26: 677-688.

Tendolkar I, van Beek M, van Oostrom I, Mulder M, Janzing J, Voshaar RO et al (2013). Electroconvulsive therapy increases hippocampal and amygdala volume in therapy refractory depression: a longitudinal pilot study. Psychiatry Res 214: 197-203.

Tisdall MD, Hess AT, Reuter M, Meintjes EM, Fischl B, van der Kouwe AJ (2012). Volumetric navigators for prospective motion correction and selective reacquisition in neuroanatomical MRI. Magn Reson Med 68: 389-399.

Trivedi MH, Rush AJ, Wisniewski SR, Nierenberg AA, Warden D, Ritz L et al (2006). Evaluation of outcomes with citalopram for depression using measurement-based care in STAR ${ }^{\star} \mathrm{D}$ : implications for clinical practice. Am J Psychiatry 163: 28-40.

Utter AA, Basso MA (2008). The basal ganglia: an overview of circuits and function. Neurosci Biobehav Rev 32: 333-342.

Videbech P (1997). MRI findings in patients with affective disorder: a meta-analysis. Acta Psychiatr Scand 96: 157-168.

Wennstrom M, Hellsten J, Ekstrand J, Lindgren H, Tingstrom A (2006). Corticosterone-induced inhibition of gliogenesis in rat hippocampus is counteracted by electroconvulsive seizures. Biol Psychiatry 59: 178-186.

Zhang WN, Chang SH, Guo LY, Zhang KL, Wang J (2013). The neural correlates of reward-related processing in major depressive disorder: a meta-analysis of functional magnetic resonance imaging studies. J Affect Disord 151: 531-539. 